\title{
Successive discrimination reversal performance as a function of level of drive and incentive'
}

\author{
JEROME M. FELDMAN, University of New Mexico. \\ Albuquerque. N. M. $\$ 7106$
}

The purpose of the present investigation was to assess the roles of drive and incentive upon the successive discrimination reversal performance of rats. Two levels of drive. high and low, and two values of incentive, large and small, were imposed upon four groups of Ss in a factorial design. All training and measurement were conducted in a simultancous discrimination General Testing Apparatus. An overall analysis of variance indicated significant main effects of incentive and problems. The results were interpreted as supporting the hypothesis that incentive exerts a significant and persistent effect upon reversal set performance in rats.

In recent years successive discrimination reversal (SDR) methodology has emerged as a sensitive technique for the assessment of interspecies performance differences (Bitterman, 1965; Warren, 1965). However, the role of experimental performance variables in influencing intraspecies differences has received less attention. Experimental investigations using SDR methodology have examined the role of two potentially potent sources of intraspecies performance differences; level of drive and magnitude of reward. In a study by Gossette \& Hood (1968) it was found that a relatively large magnitude of reward facilitated overall spatial SDR performance of pigeons. In general, the role of high drive or deprivation level was to retard discrimination performance only during the first reversal of 23 subsequent reversals. A study by Gossette \& Feldman (1968), manipulating only level of drive, has indicated that high drive retards overall spatial reversal performance in squirrel monkeys under conditions of relatively small magnitude of reward.

Investigations employing learning set methodology with subhuman primates have, for the most part, indicated no significant effects of drive or magnitude of reward level upon discrimination performance (Meyer, 1951; Miles, 1959: Schrier, 1958). However, a study by Schrier \& Harlow (1956) did indicate that learning set performance, in terms of response shift errors, was a function of magnitude of reward when each $S$ had an opportunity to experience all levels of reward within the course of learning set formation.

In studies involving the establishment of a discriminative habit in rats and then a single reversal of the reinforcement contingencies, the general finding has been that large magnitudes of reward facilitate discrimination and reversal acquisition (Hill, Cotton, \& Clayton, 1962: Hooper, 1967). In investigations manipulating drive level alone, results have been variable; high drive sometimes facilitates and sometimes retards discrimination and/or reversal acquisition (Armus, 1958; Buchwald \& Yamaguchi, 1955; Eisman, Asinow, \& Maltzman, 1956: Kendler \& Lachman, 1958; Wike, Blocher, \& Knowles, 1963).

Although most studies have indicated that rats exhibit a progressive reduction in errors in a SDR task (North, 1950; Pubols, 1957; Stretch, McGonigle, \& Rodger, 1963), the role of drive and magnitude of reward variables upon discrimination performance has yet to be studied. It was the purpose of the present investigation to assess the role of these two experimental variables upon the performance of rats in a spatial SDR task.

\section{Subjects}

\section{METHOD}

The Ss were 14 experimentally naive male blackhood rats from the colony maintained by the University of New Mexico Department of
Psychology. All Ss were approximately 120 days old at the commencement of the experimental procedure. Ss were housed individually. Apparatus

Discrimination reversal training for all Ss was conducted in a General Testing Apparatus adjoined to a metal grid animal cage where each $S$ was confined. A guillotine door separated $\mathrm{S}$ from a stimulus block platform containing two round reinforcement foodwells. Access to each foodwell was made possible through the raising of the guillotine door and a response made by $S$ of pushing back one of two transparent Plexiglas windows hinged from above. Two round, white, stimulus blocks, each 1 t/2 in. in diam and $1 / 4$ in. thick, covered the two reinforcement foodwells. In essence, the apparatus was a modification of the Wisconsin General Testing Apparatus scaled down in size so as to be appropriate for testing with small mammals An exhaust fan in the testroom provided both ventilation and a masking noise.

Design

The 14 Ss were randomly assigned to one of four groups. Six Ss and eight Ss were assigned to a low magnitude of reward group (one 45-mg Noyes rat pellet) and a high magnitude of reward group (three 45-mg Noyes rat pellets), respectively. Half of the Ss within each group were assigned to a high drive ( $70 \%$ ad libitum body weight) or low drive ( $90 \%$ ad libitum body weight) subgroup. Thus, the four groups represented were high drive/high magnitude of reward (HD-HK), high drive/low magnitude of reward (HD-LK), low drive/high magnitude of reward (LD-HK), and low drive/low magnitude of reward (LD-LK).

Procedure

The experiment consisted of three principle phases: deprivation habituation, stimulus block shaping, and reversal measurement.

Habituation to food deprivation was carried out for 14 consecutive days. During this period, food consumption for each $S$ was confined to a $2-h$ interval each day. All Ss were maintained at their assigned deprivation weights for a minimum of five days prior to the first testing phase of the experiment.

After the assigned weights of Ss were attained, a second phase of the experimental procedure was commenced wherein each $S$ was shaped and trained to push the Plexiglas windows in the test apparatus so as to dislodge stimulus blocks that covered the reinforcement foodwells. During this phase of training both foodwells contained appropriate reward values for each S. The procedure was such that with the guillotine door down, reinforcement was placed in both foodwells. The guillotine door was then raised permitting $S$ to respond and obtain reinforcement from both foodwells. Twenty such discrete trials were given each day for seven days. Following this phase of training, position preference was determined in four days by permitting $S$ to make only one reinforced response per trial. The nonpreferred position on the fourth day of testing was then selected as the reinforced position $\left(S^{D}\right)$ for the first spatial discrimination problem.

The final phase of the experiment, reversal measurement, required the initial establishment of a spatial discriminative habit. Daily 20-trial sessions were continued until a criterion of 18 correct responses during a given session had been achieved. On the following day the reinforcement contingencies were reversed, with reinforcement under the previously nonreinforced stimulus block position. Training continued until criterion performance was again established. In this manner, by successively reversing the reinforcement contingencies from left to right and right to left, etc., 19 reversals were obtained. An intertrial interval of $20 \mathrm{sec}$ and a noncorrection procedure were employed. The principle measure of reversal performance was errors per problem (reversal).

\section{RESULTS}

The results indicate that following a peak error score on the first reversal, errors progressively declined across problems to an asymptotic value well below that of the original discrimination problem. Statistical treatment by an overall analysis of variance revealed that the source of variation due to problems exerted a significant effect upon error $\operatorname{scores}(p<.01)$.

Figure 1 shows the mean error per problem score for all four groups. The major score of intergroup difference appears to be largely a function of difference in magnitude of reward $(\mathrm{p}<.02)$. The analysis of variance also indicated that all within-S interactions involving magnitude of reward were significant $(p<.01)$.

In order to assess the effects of drive and magnitude of reward levels 


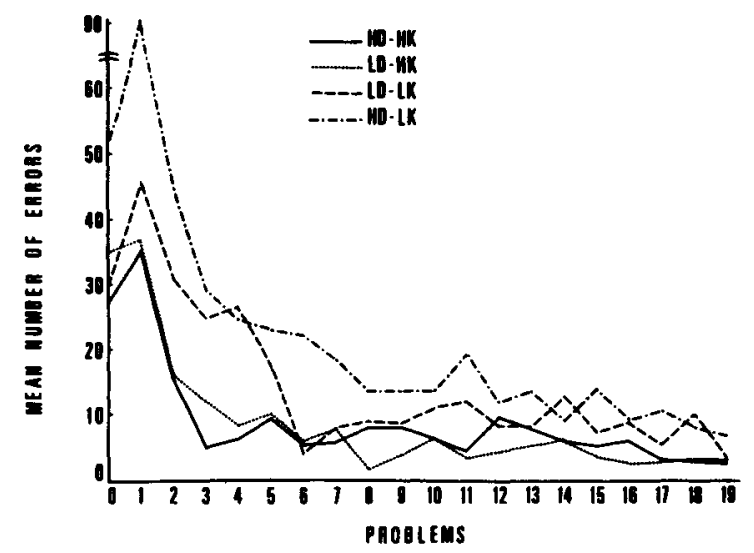

Fig. 1. SDR performance of rats as a function of drive and reward.

upon error scores when performance appeared asymptotic, a second analysis of variance was performed upon the error scores of the last 10 reversals. Results indicated that only magnitude of reward exerted a significant effect upon reversal performance $(p<.05)$.

\section{DISCUSSION}

The results of the present study appear to indicate that magnitude of reward or incentive exerts a persistent and important function upon progressive error reduction in a spatial SDR task. Although the role of drive as a source of variation in reversal performance did not achieve significance in the overall statistical analysis, it appears that it does contribute to performance differences in the original discrimination problem and first reversal of the spatial learning task.

The findings of this study are also in agreement with the research reported by Gossette \& Hood (1968) in indicating that high magnitude of reward facilitates reversal-set performance. In general, the results also support findings of studies using rats as $\mathrm{Ss}$ wherein discrimination and single reversal acquisition are an increasing function of increases in magnitude of reward (Hill, Cotton, \& Clayton, 1962; Hooper, 1967).

Results of studies involving variation in drive level upon original discrimination and single reversal performance, as reported previously, are generally not in agreement with each other (Armus, 1958; Buchwald \& Yamaguchi, 1955; Eisman, Asinow, \& Maltzman, 1956; Kendler \& Lachman, 1958; Wike, Blocher, \& Knowles, 1963). It appears, however, that these disparate results may become interpretable by positing the tentative hypothesis that the effect of drive level upon the performance of an original discrimination or single reversal problem is a function of the magnitude of reward employed. Inspection of Fig. 1 suggests that when reward level is high, there is no differential effect of drive upon performance. However, when reward level is low, high drive initially retards the acquisition and single reversal of a discriminative habit.
In general, the finding that overall reversal-set performance is affected by magnitude of reward conditions suggests that reward level does operate on some superordinate habit commonly referred to as reversal-set. Whether or not asymptotic performance differences continue to remain a function of reward level is indeterminate here.

\section{REFERENCES}

ARMUS, H. L. Drive level and habit reversal. Psychological Reports, 1958, 4, 31-34.

BITTERMAN, M. E. The evolution of intelligence. Scientific American, $1965,212,92-99$.

BUCHWALD, A. M., \& YAMAGUCHI, H. G. The effect of change in drive level on habit reversal. Journal of Experimental Psychology, $1955,50,265-268$.

EISMAN, E., ASINOW, A., \& MALTZMAN, I. Habit strength as a function of drive on a brightness discrimination problem. Journal of Experimental Psychology, 1956, 52, 58-64.

GOSSETTE, R. L., \& FELDMAN, J. M. An examination of the sensitivity of successive discrimination reversal (SDR) measures to differences in motivational level with squirrel monkeys. Psychonomic Science, 1968, 11, 157-158.

GOSSETTE, R. L., \& HOOD, P. Successive discrimination reversal measures as a function of variation of motivational and incentive levels. Perceptual \& Motor Skills, 1968, 26, 47-52.

HILL, W. F., COTTON, J. W., \& CLAYTON, K. N. Effect of magnitude of reward, percentage of reinforcement, and training method on acquisition and reversal in a T-maze. Journal of Experimental Psychology, 1962, 64, 81-86.

HOOPER, R. Variables controlling the overlearning reversal effect. Journal of Experimental Psychology, 1967, 73, 612-619.

KENDLER, H., \& LACHMAN, R. Habit reversal as a function of schedule of reinforcement and drive strength. Journal of Experimental Psychology, 1958, 55, 584-591.

MEYER, D. R. Food deprivation and discrimination reversal learning by monkeys. Journal of Experimental Psychology, 1951, 41, 10-16.

MILES, R. C. Discrimination .reversal learning in a Squirrel monkey as a function of deprivation and problem difficulty. Journal of Experimental Psychology, 1959, 57, 15-19.

NORTH, A. J. Improvement in successive discrimination reversals. Journal of Comparative \& Physiological Psychology, 1950, 43, $442-460$.

PUBOLS, B. H. Successive discrimination reversal in the white rat: A comparison of two procedures. Journal of Comparative \& Physiological Psychology, 1957, 50, 319-32.

SCHRIER, A. M. Comparison of two methods of investigating the effect of amount of reward on performance. Journal of Comparative \& Physiological Psychology, 1958, 51, 725.

SCHRIER, A. M., \& HARLOW, H. F. Effect of amount of incentive on discrimination learning by monkeys. Journal of Comparative \& Phy siological Psychology, 1956, 49, 117-122.

STRETCH, R. G., McGONIGLE, D., \& RODGER, R. S. Serial position-reversal learning in the rat: A preliminary analysis of training criteria. Journal of Comparative \& Physiological Psychology, 1963, 56, 719-722.

WARREN, J. M. Primate learning in comparative perspective. In A. M. Schrier, H. F. Harlow, and F. Stollnitz (Eds.), Behavior of nonhuman primates. New York: Academic Press, Inc., 1965. Pp. 249-281.

WIKE, E. L., BLOCHER, O., \& KNOWLES, J. Effects of drive level and turning preference on selective learning and habit reversal. Journal of Comparative \& Physiological Psychology, 1963, 56, 696-699.

\section{NOTE}

1. This research was supported in part by NSF Grant 281-044-11 awarded to Frank A. Logan. 